\title{
Acupuncture for General Veterinary Practice
}

\author{
Wing-Wah $\mathrm{CHAN}^{1)}$, Kuang-Yang $\mathrm{CHEN}^{2)}$, Hanru LIU ${ }^{3)}$, Leang-Shin WU ${ }^{1)}$ and Jen-Hsou LIN ${ }^{1) *}$ \\ ${ }^{1)}$ Departments of Animal Science and ${ }^{2)}$ Veterinary Medicine, National Taiwan University, Taipei 106, China, and ${ }^{3)}$ Department of \\ Veterinary Medicine, South China Agricultural University, Guangzhou 510642, China
}

(Received 11 April 2001/Accepted 4 June 2001)

\begin{abstract}
Since the International Society of Veterinary Acupuncture (IVAS) was founded in 1974, acupuncture (AP) has received greater acceptance by veterinary professionals throughout the world. This article introduces some important animal diseases that respond well to AP therapy. These include resuscitation of small animals, treatment of anoestrous gilts and sows, bovine reproductive disease, canine vertebral problems and equine backpain, etc. Conventional medicine considers these to be difficult cases to treat. Veterinarians have become more aware of the benefits of AP especially for those diseases, thanks to the efforts of experienced practitioners and scientists, and the many published reports on veterinary AP that have introduced some good indications for AP therapy in veterinary practice. Possible mechanisms behind the effectiveness of AP are discussed. This article aims to introduce veterinarians to good indications for AP to initiate their interest in the practice of AP. Although this is a rapidly expanding field, a long march must begin with one step. We wish this article to be the shoes for such a march. For more information on veterinary AP, contact IVAS <ivasoffice@aol.com>, P.O. Box 1478, Longmont, CO., 80502, USA (http://www.ivas.org), or your national veterinary acupuncture society (http://www.komvet.at/ ivadkom/vapsocs.htm).
\end{abstract}

KEY WORDS: acupuncture, analgesia, anoestrus, lameness, resuscitation.

J. Vet. Med. Sci. 63(10): 1057-1062, 2001

The work of many scientists and clinicians over the past 20 years has confirmed the therapeutic value of acupuncture (AP) in treating animal diseases. Increasing numbers of veterinarians wish to integrate AP therapy into their routine practice. Therefore this review paper is designed to give some clinical indications that have shown good response to veterinary AP [14, 17, 24, 33].

AP has been used in animal and human medicine for thousands of years in China [33]. In the West, it has been used in both large and small animal practices for the last three decades [14, 24]. Many published reports on AP lack rigorous controls or statistical analysis and do not stand up to rigorous scientific evaluation. However, even when these limitations are considered, many of the clinical reports provide useful information for the use of AP in routine practice. Today, the art of AP treatment has combined modern medical techniques and ancient Chinese philosophy to improve the clinical results. Some Chinese [33] and western books $[14,24]$ have documented the methods of therapy and theories of veterinary AP.

Although veterinary AP has been used to treat animal diseases for thousands of years, its actual therapeutic value was not recognised fully until the past 20 years. In 1974, the International Society of Veterinary Acupuncture (IVAS) was founded in America. Based on its aims and efforts, IVAS soon becomes the major international force to develop veterinary training in, and clinical use of, AP in animals. Today, veterinary AP is a rapidly growing part in animal medicine worldwide. The era of poor quality empirical reports has given way to an era that demands more critical reports, due to the growing need to update and enlarge our

* Correspondence to: Prof. Lin, J.-H., Department of Animal Science, National Taiwan University, Taipei 106, Taiwan, R.O.C. knowledge in the scientific basics and AP techniques. However, ironically in the Australia-Pacific regions, most veterinarians still have no chance to experience at firsthand the benefits of AP.

This is why we want to introduce you to some good indications that respond to AP. You may try to apply AP in selected conditions in your clinics. Good indications include resuscitation of emergency cases, bovine reproductive disorders, repeat breeders, anoestrus and silent heat of sows and gilts, canine paralysis and lameness as well as equine backpain $[4,16,24,31,33]$, etc. Also, without using other analgesic drugs, AP analgesia can be successful in some surgical operations in dog and cattle [20, 30]. However, AP analgesia alone is controversial. We will not mention it in detail here, but it demands great skill and deft surgery. Briefly, in circa $20-30 \%$ of animals, AP analgesia is not sufficient for absolutely painless surgery [20]. Therefore, to produce better anaesthesia for animal surgery, it is preferable to combine AP analgesia with reduced doses of modern sedation + local and / or general anaesthetics.

\section{AP THERAPY IN SOME COMMON DISEASES}

\section{Reproductive Disorders}

AP has good therapeutic effects in functional disorders of the reproductive systems of farm animals [16]. However, AP therapy has little or no clinical chance of success in patients with serious congenital defects, infections, organic pathology or neoplastic disease of the reproductive tract. Before attempting to use AP therapy in such cases, veterinarians should examine the animals in detail and consider whether or not AP is indicated. 


\subsection{Anoestrus}

Anoestrous cattle and sows are commonly encountered, especially when animals are reared under stress, such as excessive high environmental temperature and humidity, compact breeding, high stocking rates, improper management, etc. All these environmental factors can directly affect the normal function of the hypothalamic-pituitarygonadal axis (HPGA). When secretory hormones from the HPGA become imbalanced, reproductive dysfunction such as cystic follicle, persistent corpus luteum, quiescent ovaries and silent heat arise commonly. Even when the breeding environment has been improved some patients remain anoestrous, or show silent or abnormal heats. In such cases, AP can regulate the sex hormonal changes and thus restore the normal reproductive function from chronically disordered animals [14, 16, 24, 33].

The main AP points used to treat anoestrus include Baihui, GV01, GV02, BL23 and BL25. GV04 and CV04 may be used as additional points. All these points are located on the hind quarter of the body. It is easy to needle these points if the animals are restrained slightly. Experienced acupuncturists can find the AP points by anatomical determination. Less experienced clinicians may use a commercial AP point detector to locate the points. Methods used to stimulate the AP points include manual AP, aqueous $\mathrm{AP}$, electroacupuncture (EAP), laser-AP, moxibustion and moxa-AP $[8,16]$. Usually a maximum of $4-6$ AP points are selected per session and the use of more points at a given session does not seem to confer much therapeutic advantage. It is advisable to treat $1-3$ times per week. The animals are mated, or inseminated artificially, when heat is observed.

\subsection{Retained Placenta}

Retained placenta occurs commonly in cows. Known causes include Fat Cow Syndrome (excessive body condition and fatty liver at calving), twinning, age (heifers have more problems than older cows), dystocia, birth induction, deficiency of trace-elements (selenium, iodine, copper), subclinical hypocalcaemia, etc., but other aspects of its aetiology are still unknown. After delivery, smooth muscles of the uterus do not contract strongly enough to expel the placenta and lochia. Hence metritis occurs and the chance of survival decreases when placenta retains longer. AP treatment can enhance uterine contraction. AP points such as BL31-34 and Baihui are recommended [16]. Mild to strong stimulation with EAP can help to expel the retained placenta. It is advisable to treat daily until the uterine response occurs.

\subsection{Low Conception Rate}

Low bovine conception rate causes great economic loss to the dairy industry. Repeat breeders usually are treated with gonadotrophic releasing hormone [28] but the results were not so promising. Repeat breeders did not respond as well as expected when hormone treatments were given especially under a high temperature environment and a poor dairy management. A simple aqueous-AP therapy achieved a high success rate in treating repeat breeders. A dose of 10 $\mathrm{m} l$ and $5 \mathrm{~m} l$ of $50 \%$ glucose solution was injected, respectively, into Baihui and bilateral Shenpeng (Kidney Shelf, in the depression between transverse processes lumbar vertebrae 5 and 6). An overall pregnancy rate of $67 \%$ within 14 days after one treatment was reported in a preliminary trail [16]. Other methods, used by Japanese veterinarians, include moxibustion of points over the sacral area, such as BL31-BL34, bilaterally. These points gave good results in high-yielding dairy cows [8].

1.4. Impotence and Penile Paralysis

These conditions may result from overuse of the male. Boars can lose sexual interest in females. Sometimes the penis prolapses or becomes paralysed, or cannot be withdrawn into the prepuce. Penile inflammation and necrosis may occur. AP points Baihui and GV02 can improve the boar's libido and potency and restore normal mating behaviour. Manual AP or moxa-AP is suggested to stimulate the boar; he usually responds after one application. However, when the boar becomes sexually active after treatment, we emphasise the need to enforce absolute rest for 1-2 weeks before he is allowed to rejoin the breeding programme. Sometimes impotence may also result from penile paralysis, which can also be treated with manual AP or EAP. Useful points are BL31-BL33, Baihui, GV01, 02, 04 and CV01. One effective prescription is to stimulate the BL points with moxa-AP in association with EAP on Baihui and GV01 [16]. Several sessions of treatment may be needed to induce a positive response.

\subsection{Dystocia}

When parturition does not proceed normally, dystocia can be a life-threatening disorder. Surgery is the first consideration to save the patient. However, AP can be tried to assist delivery in animals in which surgery can not be used. It can successfully resolve many cases of dystocia. It helps relaxation of the pelvic ligaments, dilates the cervix, and helps to co-ordinate and strengthen uterine contractions. AP was used to assist a zoo elephant from dystocia. $10 \mathrm{ml}$ of $50 \%$ glucose solution was given at BL31-BL34, bilaterally. At $8 \mathrm{hr}$ after AP, the elephant delivered a dead calf [16].

\section{Canine Paralysis and Lameness}

Veterinarians often meet cases of canine paralysis and lameness in their practice. There are many direct or indirect causes, including vertebral trauma, joint disease, infections and intoxication, intervertebral degenerative disc disease (IVDD) and vertebral neoplasia [2, 25]. Treatments include surgery, chemotherapy, supportive medication, physiotherapy and AP. In most cases, surgery is the first consideration to treat the patients. The purpose of vertebral surgery is to decompress and immobilise the damaged vertebral column and to remove fractured bone fragments and blood clots in the spinal cord. Stabilisation of the damaged vertebrae can prevent secondary complications and help neuronal regeneration [18, 26]. Laminectomy and fenestration are currently the most common surgical procedures for IVDD [7, 10, 11]. Surgery can effectively relieve the compressive force from the damaged cord and eliminate back pain and thus prevent 
further disc extrusion. After surgery, medications usually include chemotherapy, supportive care and physiotherapy to help the spinal cord recover normal function. When vertebral operation is out of consideration, non-surgical care consists of corticosteroids or non-steroidal anti-inflammatory drugs (NSAIDs) to relieve back pain and counter neuronal inflammation [23].

Joint diseases are other causes of canine paralysis and lameness [1]. Hip dysplasia (HD) is a most common genetic disorder, often found in breeds such as German shepherd. Usually both hip joints are affected and HD can be diagnosed when the dogs are young. The primary lesion involves a joint laxity that causes biomechanical imbalance and pain. The pain has a large muscular component, probably due to internal bruising from the unstable femoral head and trochanter. Lameness occurs gradually, until finally both hindlimbs become paralytic.

Degenerative joint disease (DJD) is a chronic non-inflammatory condition associated with trauma to, or lesions in, the articular cartilage. Degenerative or proliferative lesions are usually found in joint tissues. The aetiology of DJD may be due to secondary chronic injury, erosion and thickening of the articular surfaces, and chronic metabolic or nutritional disorders.

Septic joint arthritis usually results from bacterial infection [1]. This may arise from haematogenous spread of sepsis such as in bacterial endocarditis. Joint surgery may show changes of the joint matrix granulation and viscosity. Bacteria, such as Staphylococcus and Streptococcus, are isolated from the infected joints. Treatments include surgery and drug medication. To improve joint function, surgery can stabilise the laxity or luxation of the joint. Drugs such as steroids and NSAIDS can alleviate pain and inflammation. In obese dogs, dietary regulation can be used to reduce body weight and to reduce weight-stress on the joints; this can help to improve the quality of life in obese patients.

AP is an effective method to treat canine musculoskeletal dysfunction and paralysis [5, 9]. As the causes of canine paralysis are multiple and complicated, some cases (e.g. vertebral tumour, fractures, severe genetic disorders and parasitic infestation) do not respond to AP therapy. Therefore, clinicians should determine the aetiology of paralysis or lameness before trying to use AP treatment.

Two main types of AP points are used to treat the pets: (1) points transposed from human AP locations and (2) pain points (also known as trigger points or Ahshi points).

(1) Traditional Chinese veterinary texts provide information of AP points only for large animals and birds [33]. Currently almost all AP points used in small animals are transposed anatomically from their equivalent human locations [24]. The selection of AP points to treat paralytic or lame dogs depends on the aetiology and clinical findings. Paralysis and lameness usually involve a certain degree of vertebral injury. AP points located along the vertebrae, such as those located on the BL and GV Channels, are clinically selected to treat paralytic disorders. Points on the GB, ST, SI, LI, TH and other Channels may be added. For treating disorders in the cervical area and the forelimbs, AP points such as BL10 and 11, GB20 and 21, LI11 and 15, SI03 and 09 are recommended. For paralytic disorders originating in the thoracolumbar area and hindlimbs, points such as Baihui, BL23, 26 and 34, GB30 and 34, as well as ST36, usually are used. BL62 + SI03 is another classical point combination for spinal problems. Readers can see these Channels and points in human or canine AP charts for the locations in detail [24].

(2) Due to their sensitive and uncomfortable nature, pain points are used to treat many diseases. In contrast to AP points, pain points have no defined positions. Combination of AP and pain points to treat paralysed dogs gives satisfactory results in most cases $[4,5]$. Although pain points usually occur near the lesions, they sometimes arise quite far away from them. Most clinicians locate the pain points by systematic pressure palpation of the spine and the paraspinal- and limb-musculature. They may also use a needle to stimulate the skin of, or near, the affected area to locate pain points. The patient's response depends greatly on the severity of the lesion. In cases with serious and deep pain, needling of the pain point may evoke muscular contraction over a wide area; the dogs may show a violent aversive response to needling. In cases with superficial pain, needling of the pain point may evoke only a mild local muscle contraction. However, for diagnostic purposes, responses to stimulation of pain points are not always proportional to the severity of the lesions.

When presented with a paralysed dog, a veterinarian should diagnose, as accurately as possible, the location and nature of the lesion and its cause. Some pathologies are not good indications for AP. If the case is suitable for AP therapy, the acupuncturist should develop an effective therapeutic schedule that includes the support and agreement of the owner in order to get a successful outcome. Different AP methods can be used to treat paralysed and lame dogs. Manual AP and EAP are simple and effective methods. Other methods of AP stimulation include point injection and laser AP. In principle, the selection of a successful AP method depends on (a) the experience of the veterinary acupuncturist; (b) clinical condition of the patient; (c) the degree of severity of the lesion. For example, a paralysed dog with Grade 3 disc disease (intact deep pain sensation) has $>85 \%$ chance of success within circa 30 days to manual AP. In contrast, prognosis in a Grade 4 case (absent deep pain sensation) is $<34 \%$ in circa 70 days. Also, even those Grade 4 cases that respond to AP may need a combination of manual $\mathrm{AP}$ and EAP, together with a huge amount of care and nursing (catheterisation, manual emptying of the rectum, etc) by the owner [5, 9]. Strict restriction of movement is not advised during a course of AP therapy. Instead, owners should help their pets to move gently after each session. This greatly helps local circulation and minimises the adverse effects of the inflammatory response $[3,5,9,11]$.

\section{Equine Backpain}

Equine backpain is very common, especially in race- 
horses. The aetiology is not known fully. It may be primary, secondary, or as part of a generalised disorder. Chinese medicine stresses the importance of liver, spleen and kidney function in maintaining integrity of tendon, muscle and bone/joint, respectively. Primary backpain may arise due to paravertebral muscle pain, vertebral or sacroiliac lesions or misalignments, bad saddle fit, arthrotic lesions, or "kissing" dorsal spinous processes. Secondary backpain may arise from irritation referred from thoracic or abdominal organs, from "guarding" or spasm of back muscles to compensate for joint problems in the limbs, or as part of a generalised disorder (nutritional muscular dystrophy in deficiency of Vit E-Se; myositis in azoturia).

Horses present for treatment for two main reasons. (a) They are visibly lame or show other signs of pain or stiffness, or (b) their competitive performance has fallen off gradually or suddenly. In both cases, conventional therapy usually has been tried with poor result. Skilled palpation of the BL Channel points, especially the diagnostic Shu-Association Points from BL17 to BL30, evokes a violent aversive response from affected horses. Some cases may have severe lumbar, sacroiliac, sacral or gluteal area pain. Affected horses may show back rigidity or dropped back while being ridden or exercised. Pathological findings include muscular stiffness, acidosis as a result of accumulation of muscle metabolites, including lactic acid, and raised levels of muscle enzymes in the blood. When backpain becomes chronic, affected horses may become lame and show varying degrees of muscular atrophy. Also, backpain may be associated with lameness in the fore- or hind-limbs. Hindlimb lameness is often due to pain referred from thoracolumbar or lumbosacral nerves, as in human sciatica. It may arise also in pain referred from abdominal viscera, such as in a painful kidney, ovary, uterus or colon. Forelimb lameness may arise in pain referred from cervical or upper thoracic nerves or from pain in thoracic organs (lung, pericardium, heart). Thus, neck- and back-pain often manifest as lameness in the fore- or hind-limb [21, 31].

Conventional treatment of backpain includes rest, massage, swimming and medication with analgesic or antiinflammatory drugs. In general, the disease may progressively become worse, with involvement of the hip joints and defective limb movement, and shortening of the stride. If they are not treated successfully, the commercial value of affected horses may fall markedly. However, depending on the cause, nature and duration, 70-90\% of cases respond to AP therapy [21].

Different AP methods have been used to treat equine backpain [13, 21, 31, 32]. These include injection-AP, EAP, moxa-AP, manual-AP, laser-AP etc. Paravertebral pain points always can be found in backpain horses. When pain responses are found locally, both pain points and points of BL and GV. Channels are selected for treatment. If pain action responds diffusely and hip joints are involved, one may consider adding points such as GB30, 34 and Baihui. Usually, injection-AP or EAP is used at pain points. Manual-AP or moxa-AP is usually given at traditional AP points, especially if they are not painful.

Chronic backpain is mainly a muscular motor disease that may involve the longissimus thoracis and lumborum, gluteal, biceps femoris and other muscles. Occasionally, the greater trochanter of the femur may be involved. Taking all these conditions together, stimulation on the back should be strong enough because always a large area responds painfully. For example, combination of EAP and injection-AP can give general therapeutic effect for those horses suffering from chronic backpain in association with sore hip joints. Moxa-AP and laser-AP may apply for those cases with local pain response.

Some of the top experts in equine AP treat the local pain points in the back only as a last resort. Instead they treat the relevant Jing-Well (or Ting) points. These are the most distal Channel points at the coronary band. Manual-AP at the correct Ting point(s) often releases all or most of the pain points in the back within seconds. Pain points that remain after Ting point stimulation are then treated by direct stimulation, or indirectly by using other related points, such at the Mother point or Son point of classical AP and Five Phase Theory $[14,24,33]$.

During the course of AP, most experts do not prescribe rest from the training and racing schedule unless the pain is severe and non-responsive to treatment. A few, however, advise that horses should not train heavily, or race competitively, for several weeks after disappearance of the backpain [21, 31].

\section{Resuscitation}

GV26 is the most commonly used acupoint in clinical emergencies and resuscitation in humans and animals [22]. Human GV26 is located in the midline of the nasal philtrum, one third of the way from the nose to the edge of the upper lip. In animals, the point is in the centre of the horizontal line joining the lower edge of the nostrils. Strongly needling of GV26 can resuscitate $90-100 \%$ of simple apnoeic cases within 10-30 seconds, and 40-50\% with cardiac arrest when stimulation lasts for 5-10 min [10]. GV26 can resuscitate animals effectively in anaesthetic overdose, simple apnoea and cardiac and respiratory arrest. In shocked animals, strong stimulation at GV26, can activate and restore the brain's respiratory centre and cardiovascular function, respectively. Also, by enhancing cardiac output and brain perfusion, and thus raising blood pressure, GV26 has sedative effects on convulsion and mania in animals with hypovolaemic hypotension [22]. With few exceptions, the bibliography demonstrated that stimulation of GV26 has beneficial effects. Therefore it is a good first aid indication for practitioners.

\section{POSSIBLE MECHANISMS OF AP}

In the last two decades, much research has been done on the mechanisms of AP. Most research studied the functional response of the endocrine system and the central nervous system (CNS) after AP stimulation [24, 33]. 
Lin et al. [15] studied the changes of plasma concentrations of luteinizing hormone (LH), follicle-stimulating hormone (FSH), oestradiol, progesterone and cortisol in sows after EAP treatment. They found that $\mathrm{LH}$ pulsatile release fell shortly after AP stimulation. A possible explanation involves an endorphin-mediated mechanism [16]. Endorphin inhibits LH secretion, whereas naloxone induces LH surge. Many studies showed that AP can increase endorphin levels in the brain, spinal cord and blood. Therefore, it is possible that endorphins mediate the AP-induced decrease in $\mathrm{LH}$, and after the period of depression, the pituitary becomes more responsive to GnRH. If an imbalance exists in the hypothalamic-pituitary-gondal axis (HPGA), depression of LH release may allow it to regain balance by breaking a vicious cycle or providing a needed rest period [16]. A second hypothesis involves the possibility that AP has direct effects on gonadal paracrine and autocrine control of steroidogenesis by stimulating production and release of epinephrine, catecholoestrogen, oxytocin and prostaglandin. AP can increase epinephrine levels in boars' semen [3]. Though the implications of this finding are not yet clear, epinephrine and catecholoestrogen stimulate ovarian steroidogenesis [16]. Therefore, AP may affect reproductive function by paracrine and autocrine pathways, as well as by stimulation of the HPGA. However, as the biofeedback and regulatory functions of the sexual hormones are very complex, we need much more research on AP mechanisms in this area.

Melzack and Wall's gate control theory [19], can partially explain the analgesic action of AP. In turn, the hypothesis was used to explain the desensitising effect of AP on sources of peripheral pain. Also, neurotransmitters such as endorphins and enkephalins increase in the pituitary during AP analgesic response. This finding suggests another of APanalgesia related mechanism [27].

Although these two hypotheses may provide possible mechanisms for AP-treated cases such as equine chronic backpain, it is unlikely that the analgesic effect is the only therapeutic response. For instance, some neurological disorders, such as paralysis in canine IVDD, involve neuronal damage and disturbance of neuronal propagation. Recovery of normal function may at least include a reversible ability of neuronal transmission in the lesion. AP therapy may signal surviving neurones to produce different neurone growth factors (NGFs) to maintain and recover normal neuronal pathways. Recently, the biochemical characters of some AP points have been studied in dogs. Catecholamines and substance $\mathrm{P}$ were measured in AP points that had been detected on canine skin $[6,29]$. Results indicated that AP points have more nerve endings than the surroundings. Furthermore, a polymodal receptor hypothesis [12] suggested that AP points and trigger points belong to some kind of nociceptor. These nociceptors exist in different body tissues and are easily activated by AP or moxibustion. As their sensory terminals are free axon endings, once stimulated the polymodal receptors can produce AP-effect-related therapeutic results. So far these hypotheses only partially explain the actions of
AP. Clinical trials and observations suggest that AP actions involve at least one or more factors. These are: (1) specific biochemical characters of AP points, trigger points and pain points; (2) intact anatomic structures of the target organs or systems; (3) normal neuronal pathways between the CNS and the stimulated AP points, which connects by the spinal circuits and (4) activation of certain special areas that can modify/improve the function of its controlled organs and systems. In fact, the mechanisms of AP treatment are multiple and most of them correlate to its regulatory function on the neuroendocrine system as well as the brain.

\section{CONCLUSION}

In spite of the complex philosophical basis of AP, veterinarians recognise that its practical techniques used to treat animal diseases are relatively easy to learn. By integrating AP treatment into their clinical practice, they can often obtain successful results. Some "incurable" cases also recover after AP. This increases the reputation of AP and the confidence of animal owners and the public in AP therapy. Later, after more work experience, veterinarians can try to treat other difficult cases such as canine epilepsy, hip dysplasia, circulatory disorders, etc. It is likely that AP therapy will become even more acceptable and popular in the new millennium. As it gives ideal non-pharmacological care to suffering animals, this has implications for animal welfare and for the production of chemical-free human food of animal origin.

Veterinary AP is practiced more globally and its future development is more promising than ever [14, 17, 24], with increased educational opportunities in veterinary societies, you may contact to IVAS or your national veterinary society for the AP information.

ACKNOWLEDGEMENT. We thank Dr. P. A. M. Rogers (Dublin, Ireland) and Prof. S. Hara (Iwate, Japan) for their suggestions and excellent editorial assistance.

\section{REFERENCES}

1. Bennett, D. and Taylor, D. J. 1988. Bacteria infection arthritis in the dog. J. Small Anim. Pract. 29: 207-230.

2. Braund, K.G., Shores, A. and Brawner, W. R. Jr. 1990. The aetiology, pathology and pathophysiology of acute spinal cord trauma. Vet. Med. 85: 684-691.

3. Chan, W. W. 1991. Improvement of boar semen quality after electroacupuncture treatment. Doctoral Thesis. Vet. Med. Uni., Wien.

4. Chan, W. W. 1992. Treatment of paralytic disorders with electroacupuncture in small animal practice. Intl. J. Vet. Acup. 3: 4-6.

5. Chan, W. W., Lin, J. H. and Rogers P. A. M. 1996. A review of acupuncture therapy of canine paralysis and lameness. Vet. Bulletin 66: 999-1011.

6. Chan, W. W., Weissensteiner, H., Rausch, W. D., Chen, K.Y., Wu, L. S. and Lin, J. H. 1998. Comparison of substance P concentration in acupuncture points in different tissues in dogs. Amer. J. Chin. Med. 26: 13-18. 
7. Dunny, H. R. 1978. The lateral fenestration of canine thoracolumbar disc protrusions: a review of 30 cases. J. Small Anim. Pract. 19: 259-266.

8. Hosaka, T. 1998. Moxibustion on reproductive problems in cattle. pp.176-178. In: Proceedings of the 24th Annual International Congress on Veterinary Acupuncture (Lin, J.H. et al. eds.), Taiwan, ROC.

9. Janssens, L. A. A. and Rogers, P. A. M. 1989. Acupuncture versus surgery in canine thoracolumbar disc disease. Vet. Rec. 124: 283.

10. Janssens, L. A. A., Altman, S. and Rogers, P. A. M. 1979. Respiratory and cardiac arrest under general anaesthesia: treatment by acupuncture of the nasal philtrum. Vet. Rec. 105: 273-276.

11. Janssens, L. A. A. 2001. Acupuncture for thoracolumbar and cervical disk disease. pp. 193-198. In: Veterinary Acupuncture, 2nd ed. (Schoen, A. M. ed.), Mosby Inc., U.S.A.

12. Kawakita, K. 1998. Polymodal receptor hypothesis of acupuncture and moxibustion on trigger point and acupuncture points in veterinary medicine. pp. 85-97. In: Proceedings of the 24th Annual International Congress on Veterinary Acupuncture (Lin, J.H. et al. eds.), Taiwan, R.O.C.

13. Klide, A.M. and Martin, B.B. Jr. 1989. Method of stimulation acupuncture points for treatment of chronic back pain in horses. JAVMA 195: 1375-1379.

14. Kothbauer, O. and Rogers, P. A. M. 1999. Veterinary Acupuncture. Zweimuhlen Verlag, Germany.

15. Lin, J. H., Wu, L. S., Dzang, S. H., Liu, S. H. and Chan, W. W. 1992. Pituitary responsiveness to gonadotropin-releasing hormone in gilts treated with electroacupuncture. pp. 39-42. In: Proceedings of the 18th Annual International Congress on Veterinary Acupuncture, Hawaii, U.S.A.

16. Lin, J. H., Chan, W. W. and Wu, L. S. 2001. Acupuncture for reproductive disorders. pp. 261-267. In: Veterinary Acupuncture, 2nd ed. (Schoen, A. M. ed.), Mosby Inc., U.S.A.

17. Looney, A. L. 2000. Using acupuncture in veterinary practice. Vet. Med. 95: 615-629.

18. McDonald, J.W. 1999. Repairing the damaged spinal cord. Sci. Am. 280: 55-63.

19. Melzack, R. and Wall, P. D. 1965. Pain mechanisms: a new theory. Science 150: 971-979.

20. Nam, T. C., Seo, K. M. and Chang, K. H. 1998. Acupuncture anesthesia in animals. pp. 67-84. In: Proceedings of the 24th Annual International Congress on Veterinary Acupuncture (Lin, J.H. et al. eds.), Taiwan, R.O.C.
21. Rogers, P. A. M. 1999. Treatment of backpain in the horse and dog by acupuncture. In: 1st Spanish Inter-University Meeting of Acupuncture and 3rd European Meeting of Acupuncture, Zaragoza, Spain.

22. Rogers, P. A. M. and Skarda, R. 1999. Emergency acupoint Renzhong (GV26): A bibliography and review from textbook sources. pp. 83-89. In: Proceedings of the 25th Annual International Congress on Veterinary Acupuncture. Lexington, Kentucky, U.S.A.

23. Scavelli, T. D. and Schoen, A. M. 1989. Problems and complications associated with the non-surgical management of intervertebral disc disease. Problems Vet. Med. 1: 402-414.

24. Schoen, A. M. 2001. Veterinary Acupuncture, 2nd ed., Mosby Inc., U.S.A.

25. Schunk, K. L. 1992. Disorders of the spinal cord. pp. 297-300. In: Handbook of Small Animal Practice. (Morgan, R. V. ed.), New York. Churchill Livingstone.

26. Schwab, M. E. and Bartholdi, D. 1996. Degeneration and regeneration of axons in the lesioned spinal cord. Physiol. Rev. 76: $319-370$.

27. Steiss, J. E. 2001. The neurophysiologic basis of acupuncture. pp. 27-46. In: Veterinary Acupuncture, 2nd ed. (Schoen, A. M. ed.), Mosby Inc., U.S.A.

28. Stevenson, J. S., Call, E. P., Scoly, R. L. and Phatak, A. P. 1990. Double insemination and GnRH treatment of repeatbreeding dairy cattle. J. Dairy Sci. 73: 1766-1772.

29. Weissensteiner, J., Konegger, A., Rausch, W. D., Chan, W. W., Wu, L. S. and Lin, J. H. 1998. Catecholamines in acupuncture points of dogs. pp. 199-204. In: Proceedings of the 24th Annual International Congress on Veterinary Acupuncture (Lin, J.H. et al., eds.), Taiwan, R.O.C.

30. Wright, M. and McGrath, C. J. 1981. Physiologic and analgesic effects of acupuncture in the dog. JAVMA 178: 502-507.

31. Xie, H., Liu, H. and Foster, M. 1997. Equine back pain: A traditional Chinese medical review. Equine Practice 19: 2-11.

32. Yamaguchi, T. and Kastumi, A. 1998. Effect of moxa needle therapy on chronic back pain and hip pain in race horses. pp. 129-131. In: Proceedings of the 24th Annual International Congress on Veterinary Acupuncture (Lin, J.H. et al., eds.), Taiwan, R.O.C.

33. Yu, C., Lin, J. H. et al, (60 authors in total). 2000. Modern Complete Works of Traditional Chinese Veterinary Medicine. Kangshi Book Inc., Kangshi, China. (in Chinese) 